\title{
Increasing Uplink Capacity via User Cooperation Diversity
}

\author{
Andrew Sendonaris , Elza Erkip and Behnaam Aazhang \\ Dept. of Elec. and Comp. Engineering \\ Rice University, Houston, TX 77005 \\ \{sendos, elza, aaz\}orice.edu
}

\begin{abstract}
Mobile users' capacity is limited by the fact that within the duration of any given call, they experience severe variations in signal attenuation, thereby necessitating the use of some type of diversity. We are proposing a new form of diversity, whereby diversity gains are achieved via the cooperation of in-cell users. Results show that, even though the inter-user channel is noisy, cooperation leads not only to an increase in capacity but also to a more robust system, where users' achievable rates are less susceptible to channel variations.
\end{abstract}

\section{INTRODUCTION}

There is currently great demand for increasing the throughput of wireless systems, both for voice and for data transmission, and in the framework of a multiple-access communication system. Doing so involves overcoming various impairments of the wireless channel, such as multipath fading.

Within the duration of any given call, mobile users go through severe variations in signal attenuation. Diversity is a way of combating the effects of fading, by effectively transmitting or processing more than one copy of the signal. Some well-known forms of diversity are spatial diversity and temporal diversity.

We are proposing a new form of diversity, whereby diversity gains are achieved via the cooperation of in-cell users. That is, in each cell, each user has a "partner". Each of the two partners is responsible for transmitting not only their own information, but also the information of their partner, which they receive and detect. We are, in effect, attempting to achieve spatial diversity through the use of the partner's antenna; however, this is complicated by the fact that the inter-user channel is noisy.

There is no cost, in terms of transmit power, associated with transmitting to both the base station and your partner since mobile antennas are omnidirectional. However, there are two other factors that affect the required transmit power First, a user will require more power in order to send both users' information. Second, a user will require less power because of the diversity gains. It is not clear a priori which of the above two factors will be dominant.

We analyze this system in two ways. First, we perform a capacity analysis [1], and second, we study the throughput of a given transmission scheme utilizing this concept. Both analyses aid us in evaluating if there are any capacity/transmit power gains associated with the type of diversity described herein.

\section{RESUlts}

Due to space limitations, we present here a sample of results obtained via capacity analysis. Similar results were obtained for the throughput of a CDMA implementation of user cooperation. Figure 1 illustrates the capacity region under flat fading when the two users face statistically dissimilar channels towards the base station. When the delay requirements prevent us from having larger coding delays, the rates achieved are random variables rather than real numbers. We observe an outage if these random rates fall below a certain level, and can consider the probability of outage, illustrated in Figure 2, as a performance criterion.

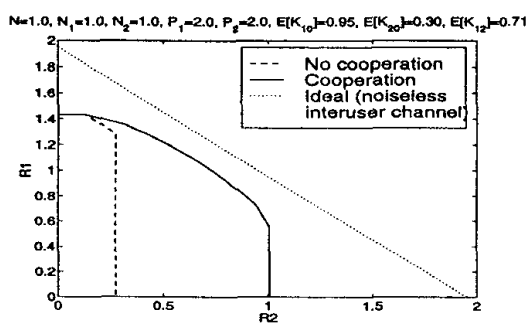

Figure 1: Capacity region.

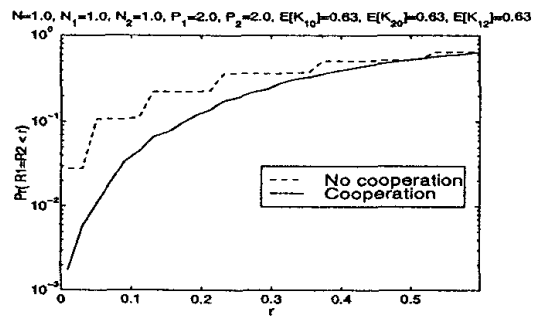

Figure 2: Probability of outage.

\section{Conclusions/Discussion}

The gains resulting from user cooperation can be substantial and are two-pronged: $A$ higher data rate and a decreased sensitivity to channel variations. Furthermore, these results hold both in an unrestricted system model, as well as when the transmission scheme is fixed.

The increased data rate with cooperation can also be translated into reduced power for the users, and thus an extended battery life. Alternatively, the cooperation gains may be used to increase cell coverage in a cellular system.

We should note that since the mobile now has to be able to detect uplink signals, implementation of the proposed system involves an increased-complexity mobile receiver. However, under certain scenarios, the benefit of an extended battery life and/or extended cell coverage will be worth the extra complexity.

\section{REFERENCES}

[1] R. Gallager, "An inequality on the capacity region of multiaccess fading channels", in Communications and CryptographyTwo Sides of One Tapestry, pp. 129-139, Kluwer, 1994. 